\title{
Patellar Instability: Traditional Surgical Interventions And A Robotic Approach
}

\author{
Serrador, Luís ${ }^{1}$; Roberto, Barbosa M. ${ }^{1}$; Santos, Bruno²; Silva, M. V. ${ }^{2}$; De Momi, Elena ${ }^{3}$; Santos, Cristina ${ }^{4}$ \\ ${ }^{1}$ University of Minho, Department of Electronic Engineering, Guimarães, Portugal, \{a68393, a68363\}@alunos.uminho.pt \\ ${ }^{2}$ Orthopaedics department, Hospital de Braga, Braga, Portugal \\ ${ }^{3}$ Bioengineering Department, Politecnico di Milano University, Milan, Italy, elena.demomi@polimi.it \\ ${ }^{4}$ University of Minho, CMEMS/DEI, Guimarães, Portugal, cristina@dei.uminho.pt
}

\begin{abstract}
Nowadays, knee complications are a common problem that can make it difficult for people to walk. One of these complications is patellar instability that can lead to recurrent patellar dislocations due to a morphological abnormality in patellofemoral joint. Due to all implications that this pathology may have for the affected person it is mandatory to treat it in order to improve the quality of life of these people. There are two principal surgeries that could be performed to treat patellar instability, depending on the severity and on the cause: trochleoplasty and tibial tubercle osteotomy. A description of the workflow of these surgeries will be presented, describing all steps and the instruments used. The main purpose of this article is to make a survey of the robotic systems that could be adapted to these interventions. This is a first approach to the application of robotic systems on these interventions. Research shows that this field of orthopedic surgery has a great potential to be improved using robotic systems. With a well-planned surgery and the development in the field of surgical robotics the duration of the surgery can be shorter as well as obtain a better result since there are precise instruments and procedures that could be adapted to perform these interventions.
\end{abstract}

Keywords - robotic-assisted surgery; trochleoplasty; osteotomy

\section{INTRODUCTION}

Patellar instability is a morphological abnormality in patellofemoral joint. This disability leads to recurrent patellar dislocations inducing some pain in the knee. This occurs principally in young and active individuals, especially females in the 2nd decade [1].

The normal patellofemoral joint has two kinds of stabilizers - active (extensor muscles) and passive stabilizer (bones and ligaments) - that are responsible to ensure the patella fixation. A modification of the anatomy of the patellofemoral joint can lead to patellar instability [1].

There are two events that characterize patellar instability. Subluxation means that the patella suffers an excessive lateral deviation without moving out totally from the trochlear groove. On the other hand, a dislocation corresponds to that cases in which the patella jumps out of the groove and it can lead to the medial patellofemoral ligament (MPFL) rupture. Recurrent subluxations may be treated with less complicated surgeries once the consequences of this kind of events are not so harmful as the patellar dislocations [2].

There are some factors leading to patellar instability such as trochlear dysplasia, patellar tilt, patella alta and an excessive tibial tubercle-trochlear groove (TT-TG) distance [1][3][4]. The MPFL rupture can be cited as a cause or a consequence, it depends if the dislocation occurs before or after the rupture. Each one of these abnormalities is treated differently and a thorough diagnostic must be done.

To diagnose these predisposing factors to patellofemoral instability a CT scan is required to visualize the patient's anatomy and evaluate some important bone structures that indicate which factor is responsible for patellar instability [1]. It is important to know that the patella only contacts with the trochlear groove from 20/30 to 90 degrees of knee flexion. When performing the CT scan, the patient's knee should be inflected about $30^{\circ}$ to ensure that the patella is contacting with the trochlear groove [1][3].

The first approach to a patellar dislocation is to treat it with a conservative method such as physical therapy and bracing. In patients with recurrent instability and trochlear dysplasia it is recommended to perform a trochleoplasty. This surgery consists in creating a new trochlear groove in order to ensure that the patella is stable and does not jumps out from the groove. In cases of patella alta and an excessive TT-TG distance it is recommended to perform a tibial tubercle osteotomy that can treat both abnormalities. In some cases, the MPFL reconstruction is performed to guarantee that the patella is stable but it is not a required intervention since most often the MPFL rupture happens when a dislocation occurs.

Nowadays, these surgeries are performed using traditional procedures but, with the advances in technology, the success of these interventions can be ensured if the surgeon is aided by some robotic systems and an optical tracking system. In orthopaedic surgeries, the preferred systems are to employ navigated surgery. In a navigated surgery, it is possible to plan the surgery using a preoperative image (e.g., computed tomography (CT), magnetic resonance imaging (MRI)) of the patient and define the tasks accurately, resulting in superior precision [5] [6].

The navigated surgery can ensure the success of the surgery as well as make them less invasive. For instance, when performing a trochleoplasty the surgeon has a small surgical field of view due to the cartilage that should remain intact. In the tibial tubercle osteotomies the cuts around the tibial tubercle should be made with high precision. These difficulties could be overcome with CAS, that includes a navigated surgery aided by a robotic system.

In this paper, the causes of patellar instability will be described and also how they can be evaluated in order to choose the approach that the surgeon must have (conservative treatment or not). Furthermore, the traditional procedures of the trochleoplasty and tibial tubercle osteotomy interventions are

* This work is supported by the FCT - Fundação para a Ciência e Tecnologia - with the reference project UID/EEA/04436/2013, by FEDER funds through the COMPETE 2020 - Programa Operacional Competitividade e Internacionalização (POCI) - with the reference project POCI-01-0145FEDER-006941. 
described. A survey regarding the robotic systems used in orthopedic surgeries is done in order to characterize the features that a robot should have to be applied in both surgeries: trochleoplasty and tibial tubercle osteotomy.

\section{CAUSES}

\section{A. Trochlear dysplasia}

Trochlear dysplasia consists in abnormal values of a set of parameters such as trochlear depth, sulcus angle, congruence angle, lateral trochlear inclination, trochlear facet asymmetry and the lateralization of the patella. These parameters provide us the severity of the deformity and it allows the surgeon to decide the best procedure [1][4]. Dysplastic trochlea is shallow, flat or even convex. In his studies, Dejour classified trochlear dysplasia in four grades based on three signs: crossing sign, supratrochlear spur and the double contour [2] [7].

This classification system is based mainly in the lateral view, although CT may assist in differentiation between types. Based on the three dysplastic signs there are four types of trochlear dysplasia:

- Type A: presence of crossing sign in the true lateral view. The trochlea is shallower than normal but is still symmetrical and concave.

- Type B: crossing sign and trochlear spur. The trochlea is flat in axial images. All the trochlea is prominent.

- Type C: presence of crossing sign and the double-contour sign on the lateral view. No prominence is seen and in axial view, the lateral facet is convex and the medial facet hypoplastic.

- Type D: combines all previously mentioned signs, which are the crossing sign, supratrochlear spur and doublecontour sign. In the axial view, clear asymmetry of the height of the facets, also referred to as cliff pattern, is evident.

\section{B. Patella Alta}

Patella alta, also known as high-riding patella, is caused by a too long patellar tendon that induces a bad position of the patella during the flexion of the knee, the patella is too high above the trochlear groove. This results in less osseous stability because the degree of flexion at which the patella engages in the trochlea is higher than in a normal knee. Furthermore, knees with patella alta have reduced patellar contact areas when compared with knees with normal patellar height [1][8].

The Caton-Deschamps index evaluates the patella height on the lateral view and it is the easiest to use and advisable for surgical planning. This index is obtained by the ratio between the distance from the lower edge of the patellar articular surface to the anterosuperior angle of the tibia outline and the length of the articular surface of the patella alta. When this ratio is greater than 1.2 indicates patella alta [1][8].

\section{TT-TG distance}

The angle between the quadriceps insertion and the patellar tendon insertion causes a laterally directed force vector. This angle is called the $\mathrm{Q}$ angle and can be measured during the physical examination tracing two lines that intersect the midpoint of the patella: one is traced from the anterior iliac spine, representing the quadriceps tension line; the other is traced from the tibial tubercle and represents the patellar tendon force line. In normal subjects it is expected a $\mathrm{Q}$ angle up to 15 or 20 degrees and it was concluded that women have the greatest values [1][3].

The tibial tubercle-trochlear groove (TT-TG) distance is a direct measure of the extensor mechanism valgus alignment previously named $\mathrm{Q}$ angle. This is achieved overlapping two slices from a CT scan. With a slice that shows the bottom of the trochlear groove and other the most proximal part of the tibial tubercle, the clinician draws two lines perpendicular to the bicondylar line that intersect the middle of the trochlear groove and the tibial tubercle. The distance between these two projections is the TT-TG value, expressed in millimeters. To conclude about the need for intervention it was established that a TT-TG distance superior to $20 \mathrm{~mm}$ is considered abnormal [1][3][9].

\section{TROCHLEOPLASTY}

As mentioned above, trochleoplasty is an intervention that aims to correct the trochlear dysplasia. The surgeon has to redefine the trochlear groove in order to perform a new groove to stabilize the patella in the sulcus. This is a complex surgery that depends of surgeon's experience [10].

For this surgery is required an extensive preoperative plan to delineate how the surgeon has to perform the cuts in the bone and how the new trochlear groove should be redefined [11].

Regarding the intervention, after all the analysis about the trochlear dysplasia in the preoperative exams, the surgeon is able to perform a trochleoplasty in order to decrease the prominence of the trochlea and create a new trochlear sulcus to avoid a patellar dislocation during all the knee movement.

The first step is to make a $4 \mathrm{~cm}$ incision using a scalpel with the knee flexed $90^{\circ}$. This allows a minimally invasive surgery and provides all the required space for the surgeon to perform the intervention [3].

The leg is then positioned in extension and a medial fullthickness skin flap is developed. The patella is briefly everted for inspection of chondral injuries and proper treatment, if needed, and then retracted laterally. Now the trochlea is exposed and changing the knee degree of flexion allows a better view of the complete operative field and it avoids extending the incision [3].

While performing the surgery, the surgeon must have an increased careful with the cartilage and the depth of bone that he will resect. The new trochlear groove must be planned to be between the articular cartilage and the medullar bone. Thus, preoperatively this zone is chosen and before resect the bone the cartilage has to be elevated.

Therefore, a drill with a depth guide set at $5 \mathrm{~mm}$ is used to remove the cancellous bone. This drill ensures the uniform thickness of the osteochondral flap and avoids injuring the cartilage or getting too close to it. Also, it keeps enough flexibility of the produced shell so that it could be modeled by the surgeon without being fractured. Once the articular cartilage is elevated from the femur, a straight osteotome is 
used to carefully create a V shape in the subchondral bone [3] [9][12]-[14].

At the end, the cartilage has to be modeled and attached to the new sulcus using some strips. These strips are attached using the distal part of the new trochlea as a starting point to the first lock and the surgeon has to be sure that this point does not contact with the patella during the knee motion. This kind of disposition of the strips ensures that the cartilage goes deep into the new trochlear groove.

\section{A. Limitations of the trochleoplasty}

During all procedures in this intervention the surgeon has to ensure that the cartilage is not damaged. This is a surgery with some special cares and one of them is the cartilage. The surgeon has to reach the bone under the cartilage and the procedures to elevate and hold them could damage it [15]. Another complication in this intervention is the field of view of the surgeon to the part of bone that is being resected due to the small incision to provide a minimal invasive surgery and due to the cartilage, that is hiding the bone that has to be removed.

Also, besides the surgical intervention aims to perform a trochlear groove of the knee as it is in the healthy knee, we need to have the conscience that is impossible to achieve a perfect trochlear groove since there are many imperfections and deformities in each patient. So far, as it is impossible to have a well-defined plan to perform during the surgery, the surgeon must check the bone removal flexing the knee to see if the trochlea is stabilized.

\section{TIBIAL TUBERCLE TRANSFER OSTEOTOMY}

There are two osteotomies that are performed to correct the patellar instability. The choice between them depends on the associated cause of patellar instability. It involves displacing the insertion of the patellar tendon in order to correct the patellar height or to realign the extensor mechanism. The medial tibial tubercle transfer is applied when we are facing a greater TT-TG distance. On the other hand, if we are facing a patella alta, a distal tibial tubercle transfer is more appropriated.

To perform this surgery, an anteromedial incision must be done once this approach is preferred instead of a lateral incision. One of the reasons is that with this incision there is no necessity to do an additional incision to perform the MFPL reconstruction, one associated problem to patellar instability, as well [16].

Regardless of the type of tibial tubercle transfer, the tibial tubercle must be completely exposed. The first step in both cases is to define the outline of the osteotomy with a scalpel in the periosteum. Next, an oscillating saw or an osteotome is used to form a 6-cm-long bone block and this must be made sufficiently deep in cancellous bone to avoid the risk of nonunion.

In medial tibial tubercle transfer the tibial tubercle is fully detached on three sides only, leaving a distal bony hinge. Thus, one single screw is enough to ensure the tubercle fixation. The pilot hole for the screw is made prior to the osteotomy with a $3.2 \mathrm{~mm}$ drill bit and is overdrilled with a $4.5 \mathrm{~mm}$ drill bit. Lastly, the tubercle is attached with a $4.5 \mathrm{~mm}$ screw.

In distal tibial tubercle transfer is required fixation with two screws once the tibial tubercle is detached completely. Before the osteotomy, the crew sites are prepared $2 \mathrm{~cm}$ apart. First the proximal portion of the tibial tubercle is pried off and while the distal portion is being cutted to the required length this portion is grasped with bone-helding forcets. The distal portion has to be as smooth as possible because any prominence would interfere with knee movement. The block is then transferred to distal position and fixation starts at the lower screw site. It is important to insert the screws perpendicular to the anterior guard of the tibia in order to avoid any movement from the tibial tubercle. The second screw is inserted when the desired amount of medialization is obtained [3][16].

\section{ROBOTIC APPROACH}

There are some difficulties that trochleoplasty and tibial tubercle osteotomy have to deal with. A robotic system could overcome these difficulties since a robotic-assisted surgery is followed by a virtual scene that allows the surgeon to know where the robot is working. With a robotic system that guides the surgeon, the surgeries could be easier to perform and could ensure their success. Nowadays, there are some robotic systems that are used in orthopedic surgeries and that could be adapted to aid the surgeons during trochleoplasty and tibial tubercle osteotomy. Some robotic systems are presented and the features that make them interesting to apply on these surgeries are described.

Mako RIO (Mako Surgical Corp.) is a hands-on robot that aids the surgeon during the knee arthroplasty. The Mako system is a semi-active tactile robotic system that requires preoperative imaging of the patient, after which a preoperative planning is performed. During the surgery, the robotic arm is under direct surgeon control and gives real-time tactile feedback during the procedure. Intraoperatively, the surgeon can virtually adjust component positioning and alignment, the knee can be moved through the range of motion and the system provides information about the alignment, component position, and balance of the soft tissue. Attached to the last part of the robotic arm is a burr that resects the bone and when the surgeon place the burr outside the pre-planned area, the burr stops and the robotic arm offers some resistance in order to prevent unnecessary and unwanted resections [17]. This robotic system has the essential features to aid the surgeon during trochleoplasty, the fact that it is hands-on allows the surgeon to positioning the cut instrument how he wants and with a preplanned zone to be resected, the robotic arm offers resistance when the instrument directs to wrong zones of the bone. Also, the virtual scene helps the surgeon seeing what he is doing since the cartilage flap above the trochlea reduces his surgical field of view.

Other robotic system that helps the surgeon intraoperatively based on a preoperative plan is the Acrobot system. The robot consists of two parts: a four-axes gross positioning system (GPS) and a three-axes active constraint motorized head that carries a high-speed rotary milling cutter. The hands-on aspect of the head is provided by a lever located at the tip of the robot near the cutter. The surgeon holds the lever and guides the cutter through the desired motions. The robot prevents of cutting outside safe regions and provides the accuracy in sculpting bones to the prescribed shape. The cutting process can be viewed on a display which also shows a $3 \mathrm{D}$ model of the knee and indicates all relevant parameters, including the 
bone remaining that must be resected [18]. The Acrobot has a similar operation as Mako RIO, and the features that make this robotic arm interesting to apply on trochleoplasty are the same: increasing the field of view using a display with the 3D positions of the instruments and the bone, allowing the surgeon to direct the cut instrument as he wants and the prevention to cut outside safe regions.

The CASPAR system (Computer Assisted Surgery Planning and Robotics) is a system that can assist orthopedic surgeons in total hip replacement, anterior cruciate ligament reconstruction and total knee replacement [19]-[21]. The CASPAR system is composed by a modified industrial robot and an interactive planning station where the preoperative plan is done based on CT images. Also, it uses a tracking system to track the leg movements during the intervention. This tracking system consists of an infrared camera and infrared passive markers that detects the excessive motions of the leg and automatically it shuts off the robot [20].

This system has some features that could be an important improvement in the trochleoplasty intervention. A robotic approach including an industrial robot to perform the cut on the bone and a tracking system could help the surgeon in the difficult task of elevating the cartilage and resecting the bone. This combined with a specific preoperative plan could improve the outcome of trochleoplasty compared to traditional procedures.

The BRIGIT (Bone Resection Instrument Guidance by Intelligent Telemanipulator) was developed by MedTech SA, a French small engineering company. This is a robot with 6 degrees of freedom mounted onto a wheeled trolley and it has a force sensor mounted. The innovation of this robot is focused in being less bulky to provide one better operation and installation of the robot and also it ensures a low price in relation to other robotic systems in orthopedic area [22]. The intervention of this robot consists on provides to the surgeon the best guide to perform the cutting process with the desired orientation. The end effector of the robot is a cutting guide and the surgeon performs the bone cutting with the surgical instrument aligned with the cutting guide [22].

The cutting guide could be an interesting method to perform the trochleoplasty and also the tubercle osteotomies above mentioned. With a thorough preoperative plan, that provides the cutting guides for the surgeon to perform the desired bone resections, the procedures could be more precise and it would not be dependent of the surgeon's experience.

\section{CONCLUSION}

Treat the patellar instability is a hard task, the surgery procedures must be carefully planned and the result must prevent new dislocations of the patella.

It is important to know where robotics could be applied and this paper shows that there are some orthopedic surgeries where robotic systems would enhance the surgeon task and thus improve the outcomes and it also elucidates about the features that these systems must have.

The difficulties associated to trochleoplasty and tibial tubercle osteotomy could be overcome with a computer assisted surgery where it is possible to track the instruments and use robotic systems with high precision, resulting in better results.

\section{REFERENCES}

[1] R. D. T. Mesquita, P. M. M. Lopes, M. Castro, and R. Cardoso, "Patellar Instability - What a Radiologist Should Know !,” Eur. Soc. Radiol., p. C2236, 2014.

[2] J. A. Feller, "Recurrent patellar instability: Assessment and decision making,” Oper. Tech. Sports Med., vol. 23, no. 2, pp. 68-76, 2015.

[3] Scott W. Norman, Surgery of the Knee. 2006.

[4] R. S. Thakkar et al., "Patellar instability: CT and MRI measurements and their correlation with internal derangement findings," Knee Surgery, Sport. Traumatol. Arthrosc., vol. 24, no. 9, pp. 3021-3028, 2016.

[5] G. Zheng e L. P. Nolte, «Computer-Assisted Orthopedic Surgery: Current State and Future Perspective.», Front. Surg., vol. 2, n. December, p. 66, 2015.

[6] M. Hoeckelmann, I. J. Rudas, P. Fiorini, F. Kirchner, e T. Haidegger, "Current capabilities and development potential in surgical robotics», Int. J. Adv. Robot. Syst., vol. 12, n. June, 2015.

[7] C. S. Dean, J. Chahla, R. S. Cruz, T. R. Cram, and R. F. LaPrade, "Patellofemoral joint reconstruction for patellar instability: Medial patellofemoral ligament reconstruction, trochleoplasty, and tibial tubercle osteotomy," Arthrosc. Tech., vol. 5, no. 1, pp. e169-e175, 2016.

[8] A. Ferreira, "Controvérsia no tratamento da instabilidade rotuliana."

[9] R. F. Laprade, T. R. Cram, E. W. James, e M. T. Rasmussen, «Trochlear Dysplasia and the Role of Trochleoplasty», vol. 33, pp. 531-545, 2014;

[10] A. E. Weber, A. Nathani, J. S. Dines, A. A. Allen, B. E. Shubin-Stein, E. A. Arendt, e A. Bedi, «An Algorithmic Approach to the Management of Recurrent Lateral Patellar Dislocation.», J. Bone Joint Surg. Am., vol. 98, n. 5, pp. 417-427, 2016.

[11] D. Dejour e P. Saggin, «The sulcus deepening trochleoplasty-the Lyon's procedure», Int. Orthop., vol. 34, n. 2 SPECIAL ISSUE, pp. 311-316, 2010.

[12] S. T. Duncan, B. S. Noehren, e C. Lattermann, «The role of trochleoplasty in patellofemoral instability», Sports Med. Arthrosc., vol. 20, n. 3, pp. 171-180, 2012.

[13] P. B. Schöttle e A. Weiler, «Trochleoplasty for Chronic Patellofemoral Instability», Oper. Tech. Orthop., vol. 17, n. 1, pp. 72-79, 2007.

[14] S. Donell e I. McNamara, «Deepening Trochleoplasty in Patellar Dislocation: Thick-Flap Technique», Oper. Tech. Sports Med., vol. 23, n. 2, pp. 129-135, 2015.

[15] S. F. Fucentese, A. Von Roll, P. P. Koch, D. R. Epari, B. Fuchs, e P. B. Schottle, «The patella morphology in trochlear dysplasia - A comparative MRI study», Knee, vol. 13, n. 2, pp. 145-150, 2006.

[16] E. Servien, P. C. Verdonk, e P. Neyret, «Tibial tuberosity transfer for episodic patellar dislocation.», Sports Med. Arthrosc., vol. 15, n. 2, pp. 61-7, 2007

[17] T. American, L. Hospital, and S. Surgery, "Robotic-Assisted Knee Arthroplasty: An Overview," no. May, 2016.

[18] B. Davies et al., "Active-constraint robotics for surgery," Proc. IEEE, vol. 94, no. 9, pp. 1696-1703, 2006.

[19] J. Schneider e W. Kalender, «Geometric Accuracy in Robot-Assisted Total Hip Replacement Surgery Biomedical PaDer Geometric Accuracy in Robot-Assisted Total Hip Replacement Surgery», vol. 9088, n. February, 2017.

[20] W. Siebert, S. Mai, R. Kober, e P. F. Heeckt, «Technique and first clinical results of robot-assisted total knee replacement», vol. 9, pp. 173-180, 2002.

[21] J. J. Frolich, P. F. Heeckt, e L. E. O. Gotzen, «Computer-Assisted Planning and Robot-Assited Surgery in Anterior Cruciate Ligament Reconstruction», Techniques, vol. 1, n. 1, pp. 50-55, 2000.

[22] P. Maillet, B. Nahum, L. Blondel, P. Poignet, e E. Dombre, «BRIGIT, a robotized tool guide for orthopedic surgery», Proc. - IEEE Int. Conf. Robot. Autom., vol. 2005, n. April, pp. 211-216, 2005. 